\title{
Dynamic Proteomics of Individual Cancer Cells in Response to a Drug
}

A. A. Cohen, ${ }^{1}+\dagger$ N. Geva-Zatorsky, ${ }^{1 *}$ E. Eden, ${ }^{1 *}$ M. Frenkel-Morgenstern, ${ }^{1}$ I. Issaeva, ${ }^{1}$ A. Sigal, ${ }^{2}$ R. Milo, ${ }^{3}$ C. Cohen-Saidon, ${ }^{1}$ Y. Liron, ${ }^{1}$ Z. Kam, ${ }^{1}$ L. Cohen, ${ }^{1}$ T. Danon, ${ }^{1}$ N. Perzov, ${ }^{1}$ U. Alon ${ }^{1}$

${ }^{1}$ Department of Molecular Cell Biology, Weizmann Institute of Science, Rehovot, Israel 76100. ${ }^{2}$ Division of Biology, California Institute of Technology, Pasadena, CA 91125, USA. ${ }^{3}$ Department of Plant Sciences, Weizmann Institute of Science, Rehovot, Israel 76100.

*These authors contributed equally to this work.

†To whom correspondence should be addressed. E-mail: ariel.cohen@weizmann.ac.il

Why do seemingly identical cells respond differently to a drug? To address this, we studied the dynamics and variability of the protein response of human cancer cells to a chemotherapy drug, camptothecin. We present a dynamic-proteomics approach that measures the levels and locations of nearly 1000 different endogenously tagged proteins in individual living cells at high temporal resolution. All cells show rapid translocation of proteins specific to the drug mechanism, including the drug target (topoisomerase-1), and slower, wide-ranging temporal waves of protein degradation and accumulation. However, the cells differ in the behavior of a subset of proteins. We identify proteins whose dynamics differ widely between cells, in a way that corresponds to the outcomes-cell death or survival. This opens the way to understanding molecular responses to drugs in individual cells.

The state of a cell is largely determined by the levels of thousands of proteins in space and time (1-4). To affect the cell state, drugs are used (5-7), but little is known about the detailed effects of drugs on the dynamics of proteins in individual human cells. Here, we ask how a drug affects the dynamics of the proteome and how these dynamics differ for individual cells. To address this, our model system was human cancer cells responding to an anticancer drug with a well-characterized target and mechanism of action, camptothecin (CPT). This drug is a topoisomerase-1 (TOP1) poison with no other known targets. CPT locks TOP1 in a complex with the DNA, causing DNA breaks and inhibiting transcription, which eventually causes cell death (8).

To follow the response to the drug, we endeavored to accurately measure the level and localization of about 1000 proteins in individual cells over time. We found a diverse protein response to the drug, with rapid localization changes of proteins specific to the drug mechanism of action, followed by slower wide-ranging temporal changes of protein levels. Furthermore, we found that the drug target TOP1 is among the very first to respond both in protein level and localization. For most proteins, the response to the drug shows moderate cell-cell variability. Deviating from this norm is a set of proteins for which responses are widely different between individual cells. Some of these proteins are involved in cell fate decisions and at least two proteins show cell-to-cell differences that are correlated with the fate of the cell. Thus, examining spatiotemporal proteome dynamics in individual cells offers clues about what is special about the subpopulation of cells that escapes the drug action.

Dynamic proteomics system. We used a retrovirus-based approach, called "CD tagging," in human H1299 lung carcinoma cells (9-14). We constructed a library of over 1200 cell clones, each expressing a different fluorescently tagged, full-length protein from its endogenous chromosomal location. Time-lapse fluorescence microscopy was used to obtain movies of the proteins over several days of growth (15).

Obtaining quantitative information from time-lapse fluorescent movies is known to be challenging, because it is difficult to automatically detect the cell boundaries and track them over time (16-20). Here, we overcame this problem by a tagging strategy that made cells more easily identifiable by image-analysis software. We used two rounds of CD tagging with the red fluorescent protein mCherry to obtain a cell clone with red fluorescence in the cytoplasm and stronger red fluorescence in the nucleus (Fig. 1A). Custom software (15) used the red fluorescence pattern to automatically distinguish the cell from its background and to differentiate the nucleus from the cytoplasm (Fig. 1B). The algorithms in the software can also automatically detect morphological correlates of cell states [e.g., cell death and mitosis (15)]. We then used this clone (H1299-cherry) as a basis for our tagged protein library. We introduced an enhanced yellow fluorescent protein (eYFP or Venus) into the red-tagged cells by an additional round of CD tagging, expanded the yellow-tagged cells into clones, and identified the yellow-tagged proteins (13). Thus, the red 
tagging is the same in all cells of the library and is independent of the second yellow tag on the protein of interest.

The library includes over 1260 different tagged proteins, of which about $80 \%$ are characterized proteins and about $20 \%$ are not characterized (for a list of tagged proteins see www.dynamicproteomics.net). We excluded the proteins whose localization did not match previous reports (about onesixth of the proteins) and studied the remaining 1020 proteins. These include diverse functional categories and localization patterns including membrane, nuclear, nucleolar, cytoskeleton, Golgi, endoplasmic reticulum, and other cell locations (fig. S1).

The CD tagging method we used tends to preserve protein functionality $(13,21,22)$. Note, however, that our use of the library does not require proteins to be functional, but merely to act as reliable reporters for the dynamics and location of the endogenous proteins. To test this, we measured the dynamics of endogenous proteins using immunoblots with specific antibodies to 20 different proteins in the parental H1299-cherry cells. In $80 \%$ of the cases (16 out of 20 ), the immunoblot dynamics agreed with the fluorescence dynamics from the movies (Pearson correlation $R>0.5, P<10^{-4}$ ) (fig. S2). Immunoblots of tagged cell clones with antibodies against green fluorescent protein (GFP) further indicated that the tagged proteins are full-length fusions (table S1). As in many high-throughput methods, we recommend that, when using the library to study specific proteins, protein functionality should be tested by other means. Most proteins named below were reannotated and tested as indicated.

Assay of proteomic response to drug. Cells were grown in 12-well optical plates in an automated fluorescence microscope with autofocus and control of temperature, $\mathrm{CO}_{2}$, and humidity. Each well contained cells tagged for a different protein. After 24 hours of growth, the drug CPT was added $(10 \mu \mathrm{M})$, and cells were tracked for another 48 hours (Fig. 1C). Images in phase, red and yellow were taken every 20 min at four positions in each well. The resulting time-lapse movies had over 200 consecutive frames per protein studied, where each frame contained 10 to 40 different cells. Movies were stored and analyzed automatically (15), resulting in traces of protein level and location in each cell over time (see supporting material for sample movies).

The cells showed vigorous divisions during the 24 hours before drug addition, with a cell cycle time of $\sim 20$ hours. When the drug was added, cells showed loss of motility and growth arrest after $\sim 10$ hours and began to show cell rounding and blebbing (morphological correlates of cell death), which reached about 15\% of the cells after 36 hours (fig. S3). Cell cycle stage at the time the drug was added did not seem to influence the response to the drug, as assayed by automatic identification of cell division and cell death events (detailed in SOM text and figs. S4 and S5). In experiments in which the drug was washed away after 48 hours, a small fraction of the cells (about $10^{-4}$ ) survived to divide and form colonies after several weeks of incubation.

Day-to-day repeats starting from frozen cells showed a mean error in the eYFP fluorescent signals of up to 15\% (fig. S6). Thus, dynamic changes as small as 20 to $30 \%$ in tagged protein intensity typically can be accurately detected by using the present assay in individual cells.

\section{Temporal profiles of protein concentration. We begin} with an account of the average population level of the fluorescent intensity of each protein and then describe the individual cell behavior. We found that most (76\%) proteins showed a significant decrease in fluorescence intensity in response to the drug, on diverse time scales. A subset of proteins (7\%) showed a significant increase in intensity. The median dynamic range of this response was a 1.3-fold change in fluorescence, and the largest changes were about fivefold. Proteins showed several classes of dynamical profiles (Fig. 2, A and B, and fig. S7) (15). The present data include dynamics of about 150 uncharacterized proteins (table S4) found throughout all profiles (Fig. 2B).

Groups of functionally related proteins tended to show similar dynamics and protein localization profiles. For example, ribosomal proteins showed rapid, highly correlated degradation (Fig. 2C), which was confirmed by immunoblots (fig. S2). Proteins with slower apparent degradation include cytoskeleton components and metabolic enzymes. The timing of degradation of most cytoskeleton-associated proteins correlated with the timing of the loss of cell motility, as measured by tracking cell position over time (Fig. 2D). Proteins that rose late in the response include helicases implicated in DNA damage repair; apoptosis-related proteins, such as the Bcl2-associated proteins BAG2 and BAG3; and the programmed cell death protein PDCD5.

The drug target is among the first to respond. The total cellular fluorescence levels of the tagged drug target TOP1 decreased on a time scale of $<1$ hour, preceding almost all other responses in the present study (TOP1 is in the first $1 \%$ of responding proteins) (Fig. 2, arrow). The rate of TOP1 fluorescence decrease was CPT dose-dependent (fig. S8, A and $\mathrm{C}$ ). Immunoblots confirmed the rapid degradation of both eYFP-tagged and untagged TOP1 in response to CPT (fig. S8D), which was consistent with previous studies (23).

TOP1 also showed rapid localization changes. TOP1 is found in the nucleoli and nucleus of cells before drug addition (24). On CPT treatment, tagged TOP1 intensity in the nucleoli dropped in less than 2 min (Fig. 3A). Fluorescence accumulated in the cytoplasm on the time scale of 5 hours after CPT addition in a CPT dose-dependent manner (fig. S8, $\mathrm{B}$ and $\mathrm{E})$. Immunoblot analysis of the TOP1-tagged clones indicated that as TOP1 was degraded, a fragment of $\sim 40 \mathrm{kD}$ 
that was detectable with GFP-specific antibody accumulated (fig. S8D). This fragment was unique to the TOP1-tagged clones (none of the other eYFP-tagged proteins tested with immunoblots in this study showed such a fragment) and may represent a degradation product of YFP-tagged TOP1. Taken together, these results suggest that, on CPT treatment, TOP1 may be proteolized in the nucleus (25) and that TOP1 fragments exit the nucleus after administration of the drug. Other DNA-damaging agents, such as cisplatin and the TOP2 poison etoposide, did not show any of these effects on TOP1 (fig. S8E).

Rapid localization changes suggest nucleolar stress and DNA damage. We found that translocation events were much rarer than abundance changes (about $2 \%$ of the proteins). The translocations that occurred, however, seemed highly specific to the drug mechanisms of action.

A set of 10 proteins showed rapid localization changes after CPT treatment, with timing similar to that of the drug target (table S2). Almost all of these proteins are localized to the nucleoli. Several of these nucleolar proteins showed a reduction in nucleolar fluorescence intensity (Fig. 3B); others showed an increase followed by a return to basal level (Fig. $3 \mathrm{C})$. Corresponding changes in the nuclear intensity outside of the nucleoli suggested that these are translocation events. We find that these proteins demonstrated similar spatial dynamics in response to the transcriptional inhibitor actinomycin D (1 $\mu \mathrm{g} / \mathrm{ml})$ (fig. S9). Similar nucleolar changes have been previously found in a mass-spectrometry study that monitored the composition of nucleoli extracted from cells responding to actinomycin D (25). These results suggest that an immediate effect of CPT on the cells used in our study is transcription inhibition, which causes nucleolar stress (26).

A slower translocation event was observed in the DNA damage repair protein RPA2. This protein moved to nuclear foci, beginning at 1.5 hours after drug addition. This is a wellcharacterized response to DNA breaks $(27,28)$, the main mechanism of cell killing by CPT. The other 14 DNA damage-related proteins in the library did not show translocations, although some showed intensity changes (fig. S10)

Slow nuclear localization changes after drug addition include the oxidative stress response pathway. In addition to the rapid responses noted above, we found that about $1 \%$ of the proteins in this study showed substantial change in nuclear localization (a change greater than $20 \%$ in the cytoplasm/nuclear ratio), on time scales of several hours (Fig. 3D). Notably, two proteins in the stress response pathway to oxidative stress, thioredoxin and thioredoxin reductase 1 , showed an increase in their nuclear/cytoplasm ratio within 8 hours after drug addition (fig. S11). As nuclear levels rise, cytoplasmic levels seem to decrease proportionally, and vice versa, which suggests that translocation occurs between these two compartments. This implies an oxidative stress response to the drug on a scale of hours, as compared with the more rapid transcription-inhibition and DNA damage responses.

We also studied the responses of 10 selected proteins to other drugs at equivalently lethal doses. A closely related derivative of CPT used clinically, Irinotecan (250 $\mu \mathrm{M})$, showed dynamics and localization changes that were similar to CPT's in shape, but slightly lower in amplitude (figs. S12 and S13). In contrast, unrelated drugs such as etoposide (a TOP-2 poison, $5 \mu \mathrm{M}$ ) showed significant differences in almost every protein studied (fig. S14).

Several proteins showed behavior that differed in individual cells. The present system allows monitoring of the cell-cell variability of each protein over time. We found that, without the drug, all proteins showed moderate cell-cell variability in their fluorescence levels, with a standard deviation between cells that ranged between 10 and $60 \%$ of the mean. This variability is in accord with that previously found in microorganisms (2, 29-32) and human cells (12). Part of this variability is due to differences in the cell-cycle stage of the cells. To quantify this, we binned the cells according to the time between their last division and the time of drug addition — an in silico synchronization approach (13). We found that about $20 \%$ of the variability is due to cellcycle stage difference, and the remainder is presumably due to stochastic processes.

After drug addition, cell-cell variability increased as a function of time by about $30 \%$ on average (fig. S15). Despite the variability, the dynamic profiles within a clone were similarly shaped; nearly all cells showed profiles of fluorescence dynamics that rise and fall together (Fig. 4, A and $\mathrm{B})$.

Diverging from this norm were 24 proteins that displayed a special behavior (listed in table S3). At first, they showed the typical variability with similar dynamics in each cell. Then, between 20 and 30 hours after drug addition, the cell population began to show dramatic cell-cell differences in the dynamics of these proteins (Fig. 4, C to F). Some cells showed an increase in the fluorescence levels; other cells stayed constant or showed a decrease. Thus, these proteins seem to show bimodal dynamical behavior. These proteins include the BCL-2-associated proteins BAG2 and BAG3, calmodulin (CALM1), ribosomal protein RPS3, and others (see table S3) that are related to aspects of cell death.

RNA helicase DDX5 and replication factor RFC1 show cell-cell differences that correlated with cell fate. We asked whether the cell-cell variability of the bimodal proteins was correlated with differential response to the drug. Most bimodal proteins did not show behavior that was correlated with cell fate (for example fig. S17, C and D). Thus, the increase or decrease of protein levels in these clones was not an indicator of cell death or survival. 
Two proteins, however, showed behavior that correlated with cell death. We find that the RNA-helicase DDX5 increased markedly in cells that survive to the end of the movies (Fig. 5A). Its levels decreased in cells that underwent the morphological changes associated with cell death. Thus, the fluorescence dynamics of DDX5 are significantly correlated with the cell fate ( $P<10^{-13}$, Fig. 5A, inset).

We also found that the bimodality of DDX5 is drugspecific, as tagged DDX5 does not show bimodal behavior in response to other anticancer drugs, such as cisplatin (see fig. S16). A second protein, replication factor RFC1, showed bimodal behavior similar to that of DDX5, rising after 20 hours in cells that survive to the end of the experiment and decreasing in cells that die (fig. S17, A and B). This observation is in line with the role of RFC1 in DNA repair (33).

Following the observation that DDX5 dynamics correlate with cell fate, we asked whether it plays a functional role in the response to the drug. For this purpose, we knocked down DDX5 by means of RNA interference (RNAi), reducing tagged DDX5 intensity by $\sim 80 \%$ (fig. S18). Adding CPT to DDX5-siRNA-treated cells showed a doubling in the death rate during the first 40 hours (Fig. 5B), compared with cells treated with nonspecific RNAi or no RNAi. This suggests that DDX5 plays a functional role in the fate of cells to CPT, consistent with its suggested antiapoptotic role (34).

Discussion. This study provided a view of the response to a drug in space and time for about 1000 proteins in individual cells. Whereas most proteins showed uniform behavior in different cells, a small subset showed bimodal behaviorcell-cell variability increased sharply about a day after the drug was added. The cells thus seem to form subpopulations with distinct protein dynamics. Among these bimodal proteins, we identified an RNA helicase (DDX5) and a DNA replication factor (RFC1), whose dynamics varied widely between cells, in a way that corresponds to cell fate: They rise in cells that survive and decrease in cells that die.

Knockdown of DDX5 caused accelerated cell killing by the drug. These proteins may thus play a functional role in escape of cells from the drug action.

We find that the cells respond to the drug by broad waves of change in protein abundance and much fewer changes in protein localization. The localization changes occurred in a temporal sequence and seem to be indicative of the drug mechanism of action. Translocations of nucleolar proteins related to transcription inhibition occurred first, on the time scale of minutes, followed by translocation corresponding to DNA damage on the time scale of an hour, followed, after several hours, by translocations of oxidative stress-related proteins. These events help define a time course for the response to the drug. Notably, the drug target TOP1 was among the first to respond. Changes in protein intensity were generally slower than translocation events, which suggested a separation of time scales between regulation in space and abundance. The present approach might thus help to elucidate the mode of action of other drugs.

Understanding the human cell as a dynamical system will require viewing it on several levels, including mRNA and protein levels, modifications, and localizations, in individual living cells over time. The present approach is a step in this direction and can be enhanced by existing proteomic methods. For example, mass-spectrometry can provide a view of protein modifications on the level of cell averages $(35,36)$ that is not observable in the present approach. The present library employs tagging that preserves endogenous chromosomal context and is built to allow robust image quantification. It provides localization and dynamics for about 150 uncharacterized proteins; the library also provides a universal epitope tag (eYFP) for potential biochemical assays of these proteins $(4,37)$. The reproducibility, temporal resolution, and accuracy of the approach allow even small dynamical features to be reliably detected.

In summary, escape of cells from this anticancer drug seems to involve cell-cell variation in the dynamics of specific proteins. Cells respond to the drug by highly specific translocation events that correspond to the drug mode of action. Observation of these effects was enabled by measuring the proteome dynamics in space and time in individual cells. The present approach provides a window into human cell biology and opens the way for understanding how seemingly identical cells show different responses to signals and drugs.

\section{References and Notes}

1. K. A. Janes et al., Science 310, 1646 (2005).

2. J. R. Newman et al., Nature 441, 840 (2006).

3. A. Belle, A. Tanay, L. Bitincka, R. Shamir, E. K. O'Shea, Proc. Natl. Acad. Sci. U.S.A. 103, 13004 (2006).

4. W. K. Huh et al., Nature 425, 686 (2003).

5. Z. E. Perlman et al., Science 306, 1194 (2004).

6. P. Yeh, A. I. Tschumi, R. Kishony, Nat. Genet. 38, 489 (2006).

7. D. W. Young et al., Nat. Chem. Biol. 4, 59 (2008).

8. Y. Pommier, Nat. Rev. Cancer 6, 789 (2006).

9. J. W. Jarvik et al., Biotechniques 33, 852 (2002).

10. J. W. Jarvik, C. A. Telmer, Annu. Rev. Genet. 32, 601 (1998).

11. J. W. Jarvik, S. A. Adler, C. A. Telmer, V. Subramaniam, A. J. Lopez, Biotechniques 20, 896 (1996).

12. A. Sigal et al., Nature 444, 643 (2006).

13. A. Sigal et al., Nat. Methods 3, 525 (2006).

14. A. Sigal et al., Nat. Protocols 2, 1515 (2007).

15. Materials and methods are available as supporting material on Science Online. 
16. U. S. Eggert, T. J. Mitchison, Curr. Opin. Chem. Biol. 10, 232 (2006).

17. C. J. Echeverri, N. Perrimon, Nat. Rev. Genet. 7, 373 (2006).

18. S. G. Megason, S. E. Fraser, Cell 130, 784 (2007).

19. R. Pepperkok, J. Ellenberg, Nat. Rev. Mol. Cell Biol. 7, 690 (2006).

20. L. Trinkle-Mulcahy, A. I. Lamond, Science 318, 1402 (2007).

21. P. J. Clyne, J. S. Brotman, S. T. Sweeney, G. Davis, Genetics 165, 1433 (2003).

22. X. Morin, R. Daneman, M. Zavortink, W. Chia, Proc. Natl. Acad. Sci. U.S.A. 98, 15050 (2001).

23. S. D. Desai, L. F. Liu, D. Vazquez-Abad, P. D'Arpa, J. Biol. Chem. 272, 24159 (1997).

24. M. T. Muller, W. P. Pfund, V. B. Mehta, D. K. Trask, EMBO J. 4, 1237 (1985).

25. J. S. Andersen et al., Nature 433, 77 (2005).

26. K. Kalita, D. Makonchuk, C. Gomes, J. J. Zheng, M.

Hetman, J. Neurochem. 105, 2286 (2008).

27. R. Sakasai et al., Genes Cells 11, 237 (2006).

28. V. M. Vassin, M. S. Wold, J. A. Borowiec, Mol. Cell. Biol. 24, 1930 (2004).

29. M. Kaern, T. C. Elston, W. J. Blake, J. J. Collins, Nat. Rev. Genet. 6, 451 (2005).

30. M. B. Elowitz, A. J. Levine, E. D. Siggia, P. S. Swain, Science 297, 1183 (2002).

31. E. M. Ozbudak, M. Thattai, I. Kurtser, A. D. Grossman, A. van Oudenaarden, Nat. Genet. 31, 69 (2002).

32. A. Bar-Even et al., Nat. Genet. 38, 636 (2006).

33. E. R. Parrilla-Castellar, S. J. Arlander, L. Karnitz, DNA Repair (Amsterdam) 3, 1009 (2004).

34. L. Yang, C. Lin, S. Y. Sun, S. Zhao, Z. R. Liu, Oncogene

26, 6082 (2007).

35. E. S. Witze, W. M. Old, K. A. Resing, N. G. Ahn, Nat. Methods 4, 798 (2007).

36. R. Aebersold, M. Mann, Nature 422, 198 (2003).

37. S. Ghaemmaghami et al., Nature 425, 737 (2003).

38. We thank the Kahn Family Foundation and the Israel Science Foundation for support. We thank E. Zalckvar, J. Bar, I. Glinert, T. Shlapok, and members of the Alon lab for discussions. The work of M.F.M. and E.E. is supported by the Yeshaya Horowitz Association through the Center for Complexity Science.

\section{Supporting Online Material}

www.sciencemag.org/cgi/content/full/1160165/DC1

Materials and Methods

SOM Text

Figures S1 to S20

Tables S1 to S4

References

Movies S1 to S4
07 May 2008; accepted 31 October 2008

Published online 20 November 2008;

10.1126/science.1160165

Include this information when citing this paper.

Fig. 1. Library of tagged proteins and its image analysis. (A) The cell clone library was generated in two steps: First, a red fluorescent tag (mCherry) flanked by splice signals was introduced on a retrovirus into the genome of H1299 cells, which resulted in cells that express proteins with an internal mCherry exon. After two rounds of tagging, a cell clone was selected with a red labeling pattern that is suitable for image analysis, bright in the nucleus and weaker in the cytoplasm. This clone formed the basis for an additional round of tagging, with a yellow fluorescent tag (eYFP or Venus) as an internal exon. Individual yellow-tagged cells were sorted then expanded into clones, and the tagged protein in each clone was identified. (B) Image analysis used the red fluorescent images to automatically detect cell and nuclear boundaries and to quantify the YFP intensity at each time point. (C) Cells were grown in an incubated microscope for 24 hours under normal conditions and then for an additional 48 hours in the presence of $10 \mu \mathrm{M}$ CPT. Cells were imaged every $20 \mathrm{~min}$, and fluorescent intensity in each cell was automatically tracked. Cell divisions and morphological changes associated with cell death were automatically detected (15). Shown is a schematic of two daughter cells of the cell in the top panel. The cell labeled with the blue track shows blebbing and fragmentation typical of apoptosis. Scale bar, $45 \mu \mathrm{m}$. MLV, murine leukemia virus. LTR, long terminal repeat.

Fig. 2. Broad temporal patterns of protein fluorescence intensity in response to drug. (A) eYFP fluorescence intensities of individual cells with tagged proteins, over 48 hours after drug addition. Thin lines, individual cells; bold black lines, population averages. (B) Normalized fluorescence shows widespread waves of accumulation and decrease in intensity. Each row corresponds to one protein averaged over all cells in the movie at each time point (at least 30 cells). Proteins were clustered according to their dynamics. TOP1 is indicated by an arrow. (C) Ribosomal proteins show correlated dynamics $\left(P<10^{-3}\right)$. Cytoskeletonassociated proteins show behaviors either correlated or anticorrelated to cell motility. (D) Cell motility (mean velocity of cell center of mass) declines 10 hours after drug addition.

Fig. 3. Rapid translocations in response to the drug correspond to nucleolar stress and oxidative stress pathways. (A). Nucleolar levels of tagged TOP1 (the drug target) decrease in $<2$ min after CPT addition. Each line corresponds to a different cell. (B) Tagged proteins show a rapid decrease in nucleolar intensity and $(\mathbf{C})$ a rapid increase in 
nucleolar/nucleoplasm ratio, followed by a decrease to basal levels. (D) Localization of fluorescence (cytoplasm intensity divided by total intensity) for all tagged proteins over time following drug addition, and examples of two tagged proteins that show changes in nuclear (red line) and cytoplasmic (blue line) intensity (chaperone PFDN5 and thioredoxin reductase 1, TXNRD1).

Fig. 4. A subset of proteins displays a bimodal response at the individual cell level in response to CPT. (A and $\mathbf{B}$ ) Examples of proteins that do not show bimodal behavior, which are representative of most proteins in the study. Profiles are similarly shaped in each individual cell. Profiles rise with time (red lines) or decrease with time (blue lines) in parallel. Cell-cell variability (CV, defined as standard deviation divided by the mean of cell-cell distribution at each time point) increases slightly over time, and the distribution of slopes of fluorescence levels shows uniform behavior. (C to E) Examples of proteins that show bimodal behavior. The dynamics after about 20 hours vary between cells: Some cells show an increase in fluorescence levels (red) and other cells show a decrease (blue). This results in bimodal distributions of fluorescent intensity slopes measured in arbitrary units (A.U.). Slopes are defined as median temporal derivative of the fluorescence levels, in the interval between 24 hours after drug addition to 48 hours (or time of cell death).

Fig. 5. DDX5 shows bimodal behavior that corresponds to the fate of individual cells. (A) The RNA helicase DDX5 shows an increase in intensity in cells that survive the drug after 48 hours, and a decrease in cells that show the morphological changes associated with cell death. Heavy colored lines are cells that die, with darker colors corresponding to earlier cell death. Blue lines are cells that do not die within 48 hours after drug administration. (Inset) Cells that show the morphological correlates of cell death have significantly higher slopes of DDX5 fluorescence accumulation than cells that do not ( $t$ test, $P<10$ to 13 ). Slopes are defined as in Fig. 4. (B) Knockdown of DDX5 causes increased rate of cell death in the presence of CPT (red line). Controls include CPT added to cells treated with nonspecific RNAi (blue line) or no RNAi (green line). Cells treated with RNAi specific to DDX5 show no cell death in the absence of CPT (pink line). 
A

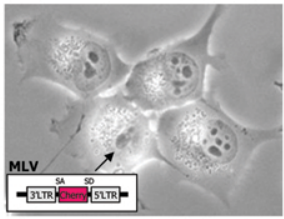

MLV RNA exoi 3LTR

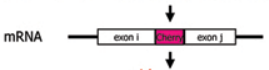
Protein
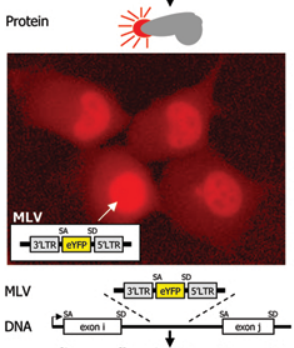

RNA evoni ILTR expe $\downarrow$

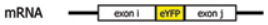
Protein

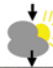

B
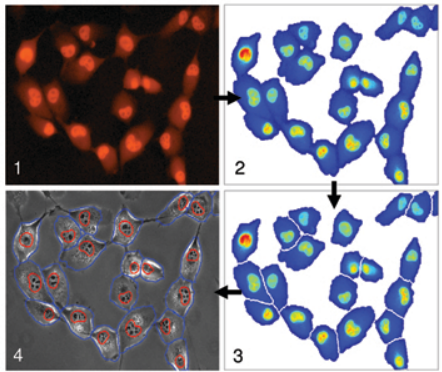

C
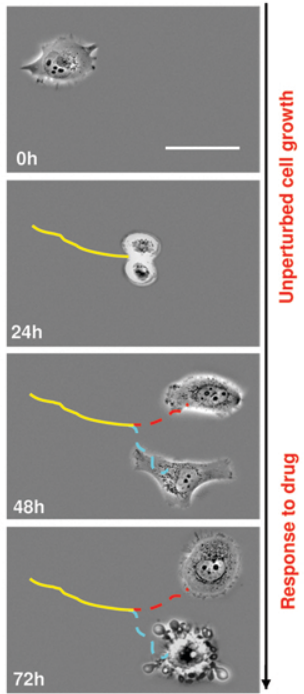
A

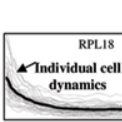

Ş
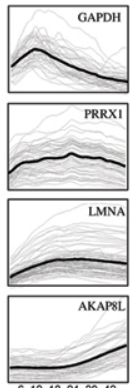

Time (hours)
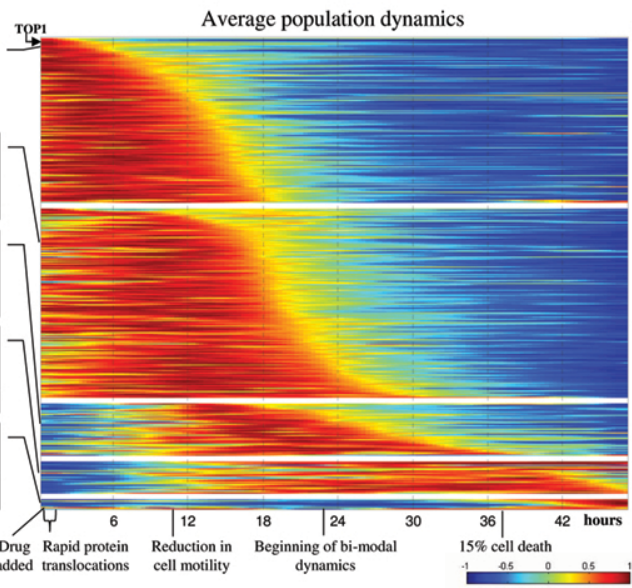

C

\section{Ribosomal proteins}
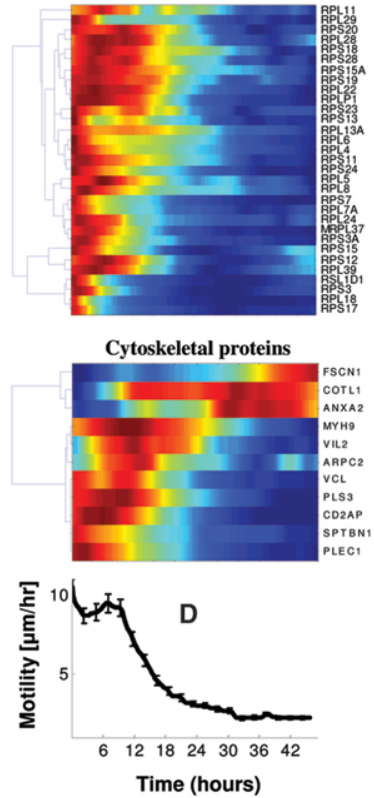
Individual cell intensity

A

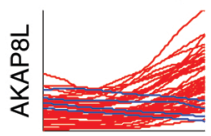

B

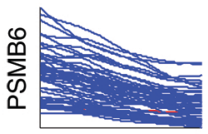

0.8

0.6

0.4
0.2

C

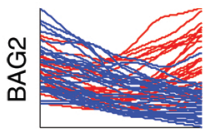

D

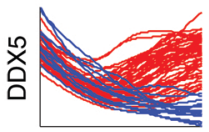

0.8

0.6

0.4

0.2

0.8
0.6
0.4
0.2

0.8
0.6
0.4
0.2

0.8

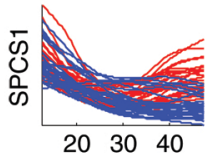

Time (hours)
Cell-cell

variability

Slope distribution
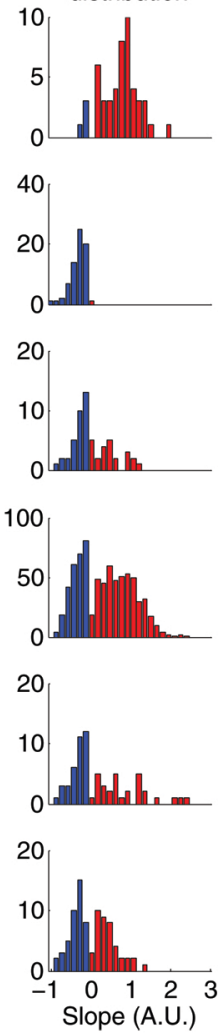
